\title{
PALEOCORRIENTES DE LAS EOLEANITAS MESOZOICAS EN EL NORTE URUGUAYO
}

\author{
Lorenzo A. Ferrando (Facultad de Agronomia, Montevideo) \\ Renato R. Andreis (NESE/UNISINOS Y FCEN, Univ. de Buenos Aires) \\ Alberto Rolon (NESE/UNISINOS)
}

RESUMEN

A consecuencia del relevamiento $1 / 100.000$ de la faja de la frontera uruguaya delimitada por la ciudad de Rivera, el paraje Capón Alto y la ruta 27, se han caracterizado las paleocorrientes de las eoleanitas Triásico Jurásicas de las unidades con que culmina la sedimentación Gondwánica de la Cuenca de Paraná en Uruguay. La formación Cuchilla Ombú de posible edad Triásico media, presenta una dirección promedio al SE; el facies é́lico de la formación Tacuarembó con una componente principal al ENE, y para la formación cuspidal Rivera, una marcada dominancia de las dirección NE.

ABSTRACT

Paleocurrents of the Triassic -Jurasic eoleanites from the final units of the Cuenca del Paraná Gondwanic sedimentation has been caracterized as result of $1 / 100.000$ survey at the uruguayan frontier belt, delimited by Rivera City, Capón Alto Place, and 27 road.

Cuchilla Ombú formation, possible aged Medium Triassic, presents SE mean orientation; eolic facies of Tacuarembó formation has principal component to ENE, and a great dominance of the NE direction at the upper Formation Rivera.

INTRODUCCION

Los resultados que se informan fueron alcanzados en el marco del Proyecto de correlación estratigráfica de la zona fronteriza entre Uruguay y Brasil entre las localidades de Rivera-Santana do Livramento y Aceguá, establecido entre la UNISINOS de Rio Grande del Sur y la Facultad de Agronomía de Uruguay financiado por la FAPERGS. 
El avance en la cartografía del sector uruguayo a escala $1 / 100.000$ en el área del Proyecto y en particular en el sector comprendido entre las ciudad de Rivera, el paraje Capón Alto y la ruta 27, ha permitido confirmar la siguiente Columna Estratigráfica en superficie, en la que se indican sus edades más probables:

$\begin{array}{ll}\text { FM. Arapey } & \text { Js }-\mathrm{Ki} \\ \text { FM. Rivera } & \mathrm{Jm} \\ \text { FM. Tacuarembó } & \text { TRs } \\ \text { FM. Cuchilla Ombú } & \text { TRm } \\ \text { FM. Buena Vista } & \text { TRi } \\ \text { FM. Yaguarí } & \text { Ps }\end{array}$

Dentro de este relevamiento se ha prestado particular interés a la caracterización de las eoleanitas que conforman las formaciones Cuchilla Ombú y Rivera, así como las que aparecen dentro de las litologías de la formación Tacuarembó. Si bien se han reconocido algunas dunas dentro de la formación Buena Vista, como su principal área de afloramiento se desarrolla hacia Rio Grande del Sur, el número de observaciones es muy escaso como para obtener validéz estadística a los datos.

\section{RESULTADOS}

La formación Cuchilla Ombú, se reconoce según la definición original de FERRANDO y ANDREIS (1986) y se integra por areniscas medias a finas y en menor proporción finas y medias; los colores pueden ser grises claros (N8) o amarillentos (5YR7/2), siendo muy frecuente en este sector de la cuenca las pigmentaciones en varias tonalidades rojizas (10R6/6, 7/4, 5/6, $5 / 4,4 / 6,3 / 4 \circ 5 \mathrm{R} 4 / 2)$.

Las psamitas presentan un predominio de estructuras cruzadas de tipo cuneiforme planar y en menor proporción artesas, acanaladas 0 arqueadas, Estas estructuras exhiben porte grande y en consecuencia los sets alcanzan comúnmente más de $5 \mathrm{mt}$.; son muy raras las cruzadas de pequeño porte.

Estas estructuras quedan definidas por estratificación centimétrica, aunque en algunos afloramientos se observan estratos más gruesos que pueden alcanzar hasta los $60 \mathrm{cms}$, siendo en este caso macizos.

Son raros los estratos que presentan convolutas o evidencias de fluidización e incluso bioturbación de tipo endichnia, estando desarrollados en la base de la Formación. 
Las caracteristicas de los litosomas evidencian claramente su origen é́lico. La orientación de las paleocorrientes muestra para las 420 medidas realizadas en el área estuaiada, (Figura 1) una relativamente amplia distribución, entre $60 \%$ y 240 el $80 \%$ de las medidas, con una moda marcada entre $90^{\circ}$ y $120^{2}(16 \%)$, aunque la media de los valores se ubica en torno a direcciones $\mathrm{SE}(1502)$, lo que coincide con las observaciones de otras áreas de afloramiento de esta Formación (FERRANDO y ANDREIS, 1986; FERRANDO et al. 1987). Las paleocorrientes $y$ las observaciones de campo han permitido definir estos cuerpos de dunas tanto como de tipo barján como seif.

Dentro del área de afloramiento de la formación Tacuarembó (FERRANDO, 1986), se han encontrado con cierta frecuencia facies de origen eólico apareciendo saltuariamente dentro de los perfiles, indicando la existencia de subambientes dentro de la cuenca de sedimentación, que no se han reconocido en el área tipo, donde se la caracterizó como fluvial.

Litológicamente predominan las psefitas finas a medias siendo escasas las variaciones hacia extremos más finos o gruesos, de colores dominantemente claros (10YR7/4, 6/6, 8/6, 5YR7/2, 4/4, 5/6 y N7).

Las observaciones realizadas muestran a las artesas como las estructuras más comunes y en menor proporción cuneiformes planares; internamente estos cuerpos están compuestos de estratos finos y láminas bien definidas por variaciones granulométricas y de color, con contactos bien definidos. Estas estructuras alcanzan sólo un desarrollo de mediano porte y en estratos que pocas veces superan los dos metros de potencia. En la parte basal de estos cuerpos es frecuente encontrar estructuras convolutas, evidencias de fluidiza-: ción de los sets e incluso bioturbaciones bien desarrolladas correspondientes a Anchorichnus cotonus.

La distribución de las paleocorrientes de acuerdo a 196 medidas, (Figura 2) muestra una muy amplia distribución de las mismas, con dos modas discretas una entre $30^{\circ}$ y $60 \%$ (16\%) y otra entre $90 \%$ y 120 (15\%) evidenciando una clara rotación de los paleovientos con respecto a la unidad inferior.

Las litologías de la formación Rivera (FERRANDO, 1986) se definen en esta zona por areniscas medias y medias a finas con menor participación de psamitas finas, de colores rojizos (10R6/6, 6/2 y 5R6/6), presentando comúnmente una débil silicificación.

Las estructuras pueden ser cuneiformes planares o artesas con raras tangenciales simples, correspondiendo a grandes cuerpos de dunas, ya que los 
sets normalmente presentan entre 4 y 7 mts. de potencia, siendo raros los cuerpos de menor tamaño.

Estas estructuras mayores quedan definidas por estratos laminados o tabulares con laminación, resaltados por discretas variaciones granulométricas, en el contenido de feldespatos o débiles cambios en la coloración. No se han observado en estas litologías evidencias de bioturbaciones o acción de aguas.

Los distintos afloramentos permiten caracterizar las dunas de la formación Rivera como de tipo barján, aunque con alguna frecuencia se observan seif, teniendo éstos en principios menores dimensiones que los primeros.

La distribución de las 416 medidas de paleocorrientes (Figura 3) muestra una distribución unipolar (entre $60 \%$ y $90 \%$ el $31 \%$ de los datos) y una dispersión perfectamente compatible con la caracterización de tipo barján que se ha realizado. Confirma además una notable variación de los paleovientos desde los tiempos Triásicos, hacia el SE en Cuchilla Ombú, hasta el Jurásico medio - superior (NE), mostrando la formación Tacuaremb6, una alternancia de ambas direcciones.

\section{CONCLUSIONES}

La realización para el área estudiada, de un número estadísticamente significativo de medidas, permite caracterizar las eoleanitas del mesozoico uruguayo de la siguiente forma:

a) para la formación Cuchilla Ombá: vientos con cierta amplitud en su distribución ( $90 \%$ a $210 \%)$, pero con una componente hacia el SE, originando dunas de gran tamaño.

b) para los facies é́licos intercaladus en las facies fluvialea de la formación Tacuarembó: gran amplitud en las direcciones (de 3609 a $180 \%$ ), con dos modas poco destacadas: una al NE ( $30 \bumpeq$ a $60 \%$ ) y otra al SE ( $90 \%$ a $120 \%$ ), formando cuerpos de mediano porte.

c) para la formación Rivera, distribución unimodal de las paleocorrientes con dirección dominante al NE (60\% a 90\%) correspondientes a grandes dunas de tipo barján en forma dominante.

\section{BIBLIOGRAFIA}

FERRANDO, L.A. (1986) Guía Excursión a los depósitos Gondwánicos de Tacuarembó Proyecto 193 Silúrico-Devónico de América Latina. IV Reunión Técnica. Tacuarembó, Uruguay. 
FERRANDO, L.A. y R.R. ANDREIS(1986) Nueva estratigrafía en el Gondwana de Uruguay. 1. Congreso Latinoamericano de Hidrocarburos. ARPEL I: 295323, Buenos Aires, Argentina.

FERRANDO, L.A. R.R. ANDREIS y J.R. MONTAÑA(1987) Estratigrafía del TriásicoJurásico uruguayo en la Cuenca de Paraná. Atas do III Simposio SulBrasileiro de Geología, 1:373-378, Curitiba, Brasil.
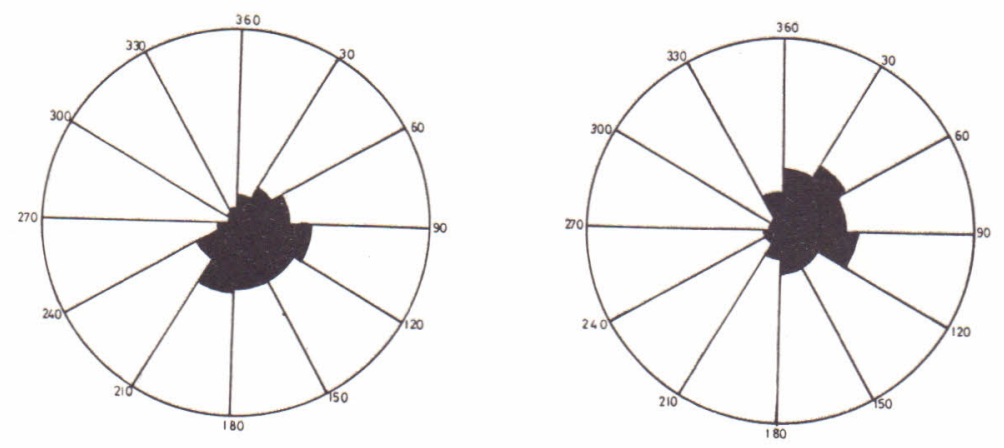

Formación CuCHILLA OMBU.

Formación TACUAREMBO

(facies eólico).

$n=420$

FIGURA 1

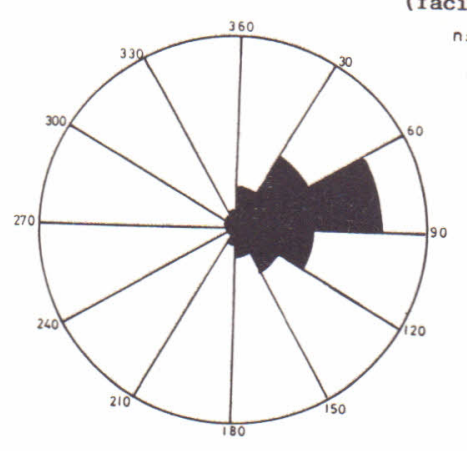

$n=196$

FIGURA 2

Formación RIVERA.

$n=416$

FIGURA 3 
